\title{
Frontal Cicatricial Alopecia: An Unusual Complication of Otoplasty
}

\author{
Yuksel Toplu* \\ Department of Otorhinolaryngology, University of Inonu, Turkey
}

Received: 制: October 08, 2018; Published: 制: October 16, 2018

*Corresponding author: Yuksel Toplu, Inonu University Medical Faculty, Otorhinolaryngology Department Malatya, Turkey

\begin{abstract}
Prominent ears are common abnormalities that can have negative effects on the psychological development of pediatric patients. An otoplasty is a frequently performed cosmetic surgery used to correct prominent ears. Although there are many otoplastic methods, all of them have some associated risks and/or complications. Here we discussed an unusual complication of otoplasty, frontal cicatricial alopecia, also called scarring alopecia, which refers to a group of rare disorders that destroy hair follicles. The follicles are then replaced with scar tissue, causing permanent hair loss. This complication was caused by a postoperative over-stretched ear bandage and has not yet been defined in the PubMed literature. Surgeons should be aware of the amount of pressure created by a bandage, as well as any possible tissue reactions from the otoplastic materials, including the bandage, Beta fix, gauze and sutures, to avoid this complication.
\end{abstract}

Keywords: Otoplasty; Bandage; Cicatricial Alopecia

\section{Introduction}

Prominent ears are the most common congenital deformities of the external ear, affecting 5\% of the population. Prominent ears may result from a combination of ear defects, including underdevelopment of the antihelix, hypertrophy or anterior rotation of the conchal cartilage, and inadequate definition of the helical rim [1]. An otoplasty is a frequently performed cosmetic surgery used to correct prominent ear deformities [2]. Ely first described otoplasty in 1881, and more than 200 otoplasty methods have been described in the literature today [2,3]. However, all of these methods have certain risks and/or complications, which can be divided into early and late complications. The previous appear up to 14 days postoperatively, and the latter occur after the initial 14-day period $[2,3]$.

Here we discussed an early complication, frontal cicatricial alopecia, caused by a postoperative over-stretched ear bandage. In the PubMed literature, this unusual otoplasty complication has not yet been defined. We believe that the appropriate surgical approach, proper bandage tension, suitable bandage material, awareness of postoperative care and surgeon's experience are significant in the prevention and healing of this complication.

\section{Case Presentation}

A 20-year old female patient presented to our clinic with complaints about hair loss (alopecia) following an otoplasty. She had been operated on in our clinic ten days previously. Before the operation, she had no complaints of hair, skin or chronic diseases, such as contact or allergic dermatitis. Upon examination, she had a horizontal alopecial region in the frontal hairy scalp, approximately $10 \mathrm{~cm}$ in length by $3 \mathrm{~cm}$ in width (Figure 1). The examination of the otoplastic auricles was completely normal. This patient underwent an otoplasty ten days previously under general anesthesia. A combination of the Furnas and Mustarde suturing techniques was used for the surgery. The auricles were covered postoperatively by gauze with antibiotic pomade and dressed in a fabricated ear bandage. The antibiotic gauze was changed daily and dressed in an auricular bandage during the first week. By the end of the week, the gauze and the bandage were removed, and the external auricles seemed quite smooth, natural and symmetrical (Figure 2). However, we observed frontal swelling, colour changes, itching, crusting and hair loss on the postoperative 10th day. A dermatology department consultation was conducted for a definitive diagnosis, and to determine the appropriate medical treatment. They suggested antibiotic (mupirocin) pomade therapy, with a diagnosis of cicatricial alopecia. The alopecial region regressed minimally due to disease stabilization by the end of the first year (Figure 3). Therefore, surgical excision and suturing were conducted by us for the treatment of the remaining alopecial region at the end of the first year (Figure 4). 


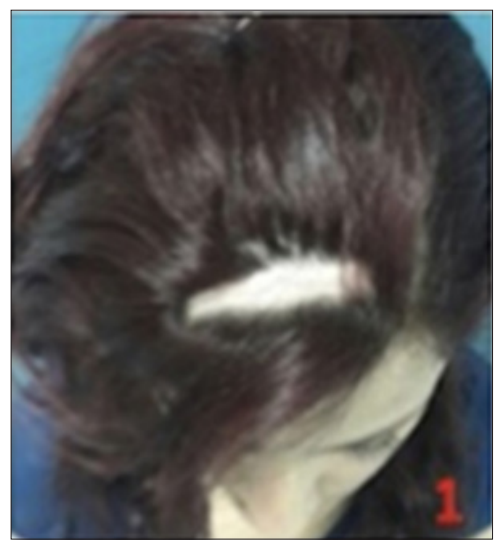

Figure 1: Images of the alopecial region in the frontal hairy scalp.

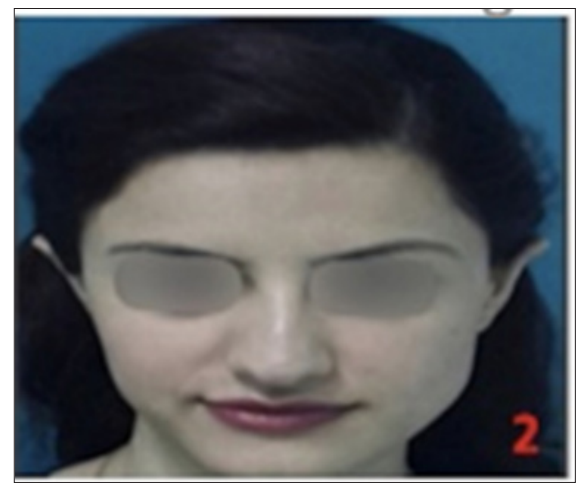

Figure 2: Images of the patient 10 days after otoplasty; external auricles seemed quite smooth, natural and symmetrical.

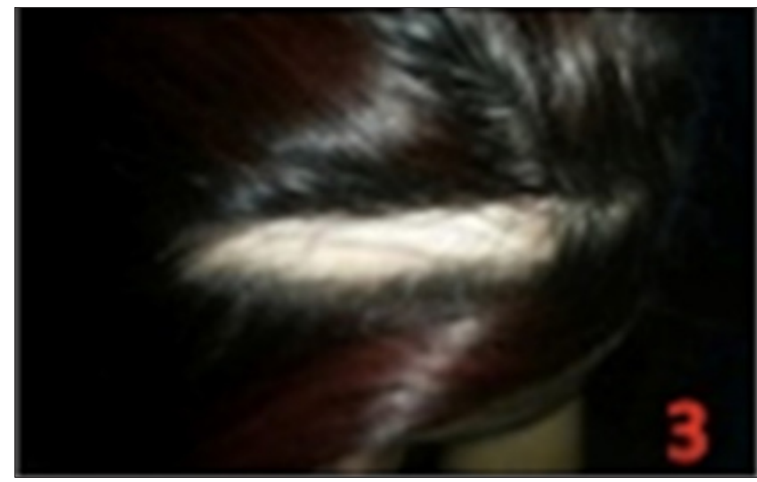

Figure 3: Images of the alopecial region regressed minimally due to disease stabilization by the end of the first year.

\section{Discussion}

All otoplastic methods have some associated risks or complications that can be divided into early and late sequelae, with the former occurring up to 14 days postoperatively and the latter occurring after the initial 14-day period [1]. The early complications include hematoma, bleeding, infection, skin reactions and wound dehiscence, while late complications include suture extrusion, hypersensitivity, asymmetry, insufficient correction, overcorrection, temporal nerve paresis, torticollis and bilateral outer ear canal stenoses [2-4]. In this paper, we discussed an unusual early complication, frontal cicatricial alopecia, due to a post-otoplasty over-stretched ear bandage. The role of postotoplasty bandaging is to avoid the accumulation of blood between the cartilage and the skin [5]. In addition, proper bandaging will support the new shape of the ears. The covering should apply just enough pressure to accomplish these; However, too much pressure can change the skin colour, disrupt the blood supply and cause oedema, ecchymosis and ulceration. Ischemia is a significant problem at this point, and the most serious effects occur within 24 to 48 hours after the application [5].

If the patient begins to complain about a tight bandage or unexpected pain, bandage complications should be considered. Proper re-examinations and bandage changes are very important. In our case, the frontal oedema, ecchymosis and ulceration could not be seen because they were hidden by bushy hair. If the oedema and ecchymosis had been detected, the early alopecia could have been prevented using cotton padding, along with close monitoring of the tissue oedema, proper bandage tension and educating the patient about appropriate wound care [5,6]. Cicatricial alopecia, also called scarring alopecia, refers to a group of uncommon disorders that destroy hair follicles, which are turned into scar tissue, causing permanent hair loss [7]. Cicatricial alopecia has two forms; in the primary form, the hair follicle is the target of the immune cells, while in the secondary form, the hair follicle is destroyed by something else, such as an infection, radiation or a tumour. However, the cause of cicatricial alopecia is not fully understood. Redness, pain and swelling occur in the area of the hair follicle. If the follicular structure and oil glands are destroyed, the hair follicle cannot grow again, and the hair is persistently lost [7].

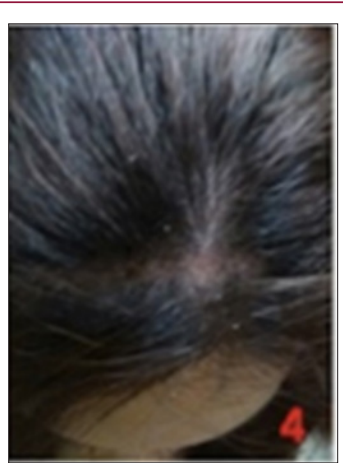

Figure 4: Images of the surgical treatment of the remaining alopecial region at the end of the first year.

A dermatologist should examine the hair follicles on the scalp and can recommend a scalp biopsy if necessary. It is important to begin treatment early because the hair loss in cicatricial alopecia can become permanent. Oral treatments may include antimalarials, antibiotics and/or immunosuppressive drugs. Topical medications applied directly to the skin include corticosteroids, topical tacrolimus and other agents that suppress or modulate the immune system. One form of this disorder is caused mainly by neutrophils and can be treated with oral and topical antibiotics and antiinflammatory medications. This is usually a long-term treatment 
[7]. After the disease stabilizes and has been inactive for 1 or 2 years, surgical hair restoration (hair transplantation or follicular micrografting) or scalp reduction (resection and suturing) may be useful for restoring the alopecial areas [6,7]. In this case, minimal improvement was seen in the alopecial region during the 12-month follow-up period with the use of the prescribed topical pomade (mupirocin) (Figure 3). Therefore, we performed surgical excision and suturing of the remaining alopecial region at the end of the first year. As a result, our case had an acceptable outcome (Figure 4).

It is known that when a proper ear dressing achieves appropriate immobilization, obliteration of dead space, compression and protection from trauma, many complications following an ear reconstruction can be avoided [5,6]. However, it is difficult to apply and maintain proper dressings because the auricle has complex contours and protrudes from the head. There are several types of commonly used ear management dressings (Figure 5), each with its own advantages and disadvantages. For example, the popular traditional mastoid dressing may be dislodged when bandaged too loosely or may cause pressure necrosis when bandaged too tightly. This bandage is difficult to apply and remove, and is sometimes bulky, heavy or uncomfortable. Moreover, fabricated bandages can cause excess moisture and discomfort if they are too thick and not air-permeable. The complication we saw in this case was likely due to this type of bandaging. In fact, we actually recommend the use of elastic bandages in otoplastic surgeries (Figure 5C). Elastic bandages are easily applied to complicated contours and can control the expansive and contractile forces.

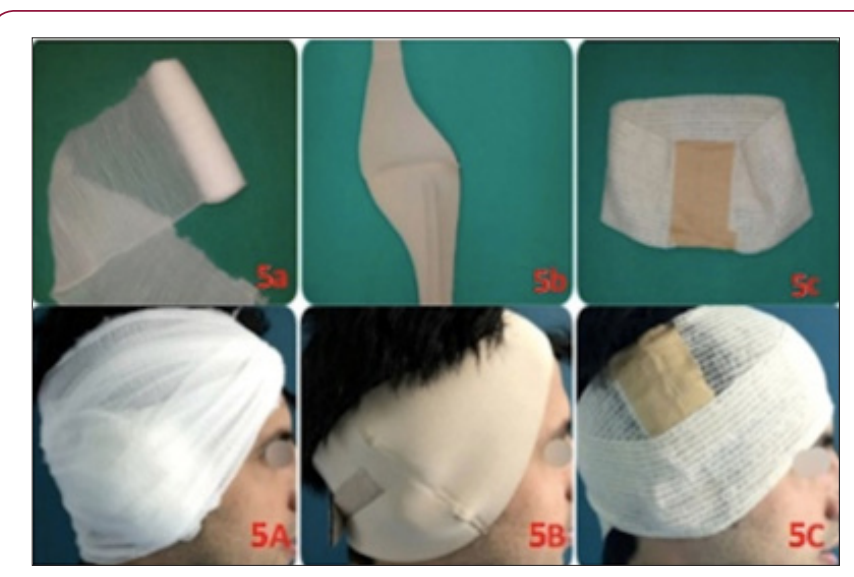

Figure 5: Images of the types of ear dressings that commonly we used after otoplasty.
In addition, these bandages can be easily and quickly prepared according to the patient's head circumference in any operating room, and the splint is light, inexpensive and easy to apply and remove. Moreover, we have not encountered any bandage complications since we began using this type of bandage. In summary, most otoplastic complications can be reduced by careful pre- and perioperative examinations (e.g. cartilage elasticity), a good surgical technique, the use of the proper bandage materials and attentive postoperative care. The surgeon should be aware of the amount of pressure the bandage applies, and possible tissue reactions due to the surgical materials, including the bandage, beta fix, gauze and sutures, to avoid complications.

\section{Declaration of interest}

The author reports no conflicts of interest. The author alone is responsible for the content and writing of the paper.

\section{References}

1. Adamson PA, Litner JA (2006) Otoplasty technique. Facial Plast Surg Clin North Am 14(2): 79-87.

2. Owsley TG, Biggerstaff TG (2009) Otoplasty complications. Oral Maxillofacial Surg Clin N Am 21(1): 105-118.

3. Limandjaja GC, Breugem CC, Mink van der Molen AB, Kon M (2009) Complications of otoplasty: a literature review. J Plast Reconstr Aesthet Surg 62(1): 19-27.

4. Toplu Y, Toplu, SA, Sapmaz E, Deliktas H (2014) An Unusual Cause of Conductive Hearing Loss: Bilateral Complete Meatal Obstruction Following Otoplasty. J Craniofac Surg 25(2): e168-170.

5. Black J, Alves P, Brindle CT, Dealey C, Santamaria N, et al. (2015) Use of wound dressings to enhance prevention of pressure ulcers caused by medical devices. International Wound Journal 12(3): 322-327.

6. Kumar B, Das A, Alagirusamy R (2014) Effect of material and structure of compression bandage on interface pressure variation over time. Phlebology 29(6): 376-385.

7. Beheshtiroy A, Hajmanoochehri F, Hossienghamar F (2015) An epidemiological study of 97 cases of primary cicatricial alopecia in Iran. Dermatology Reports 7(2): 5960. 


\section{ISSN: 2574-1241}

DOI: 10.26717/BJSTR.2018.10.001905

Yuksel Toplu. Biomed J Sci \& Tech Res

(C) (P) This work is licensed under Creative

Submission Link: https://biomedres.us/submit-manuscript.php

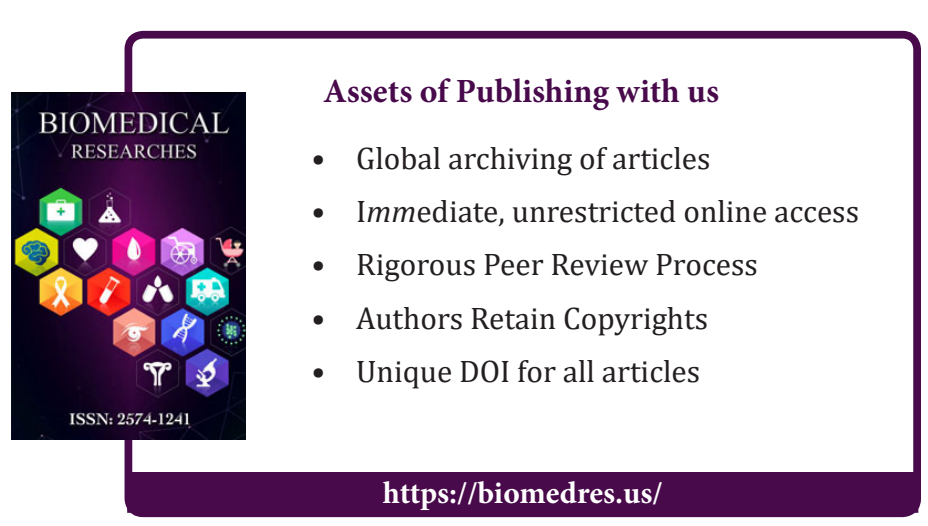

